\title{
High-resolution Anorectal Manometry for Autonomic Dysreflexia in a Patient With Incomplete Cervical Spinal Cord Injury
}

\author{
Tae Hee Lee, ${ }^{1}$ Su Jin Hong ${ }^{2}$ and Joon Seong Lee ${ }^{1 *}$ \\ ${ }^{1}$ Institute for Digestive Research, Digestive Disease Center, Soonchunhyang University Seoul Hospital, Seoul, Korea; and ${ }^{2}$ Department of \\ Internal Medicine, Soonchunhyang University College of Medicine, Bucheon, Gyeonggi-do, Korea
}

\begin{abstract}
A 48-year-old man was referred to the constipation clinic for the evaluation of defecation difficulty. He was diagnosed with incomplete spinal cord injury at the level of $\mathrm{C} 53$ years ago. On admission, he reported one bowel movement during the previous 7 days. He complained anal pain, anxiety, dyspnea and headache which were developed only during defecation episode. He also presented with pale and cold feet and numbness of lower extremities. Colonoscopy and abdominal computed tomography showed unremarkable findings. Anorectal function tests were performed for the evaluation of difficulty in defecation. He was not able to expel a rectal balloon containing $150 \mathrm{~mL}$ of water. Barium defecography showed typical findings suggestive of non-relaxing puborectalis syndrome. Total colon transit time was 68.4 hours. In the high-resolution anorectal manometry (HRARM; ManoScan, Sierra Scientific Instruments, Los Angeles, CA, USA) examination with balloon expulsion, mean resting anal pressure $(69.3 \mathrm{mmHg})$ was intact but maximum squeezing pressure $(95.4 \mathrm{mmHg}$ ) was low. During defecation, paradoxical anal contraction with inadequate propulsive force (type II pelvic floor dyssynergia; mean intrarectal pressure $7.1 \mathrm{mmHg}$, mean residual anal pressure $67.1 \mathrm{mmHg}$ ) was noted. The rectoanal in-
\end{abstract}

hibitory reflex was developed at rectal balloon dilatation of 20 $\mathrm{mL}$. Interestingly, rectal balloon inflation with more than 180 $\mathrm{mL}$ of air caused similar difficulties with defecation as well as paradoxical anal contraction (Figure A). In general, there was an increase in anal relaxation with raising degree of rectal inflation in a constipated patient (Figure B). The relief of rectal inflation resulted in the complete resolution of these symptoms. Although these findings are not specific to the autonomic dysfunction, the patient was diagnosed as autonomic dysreflexia (AD) with the help of neurologic consultation.

$\mathrm{AD}$ occurs up to $90 \%$ of people with upper thoracic (above the neurologic level of T6) and cervical spinal cord injury. ${ }^{1}$ Although it can be asymptomatic or less severe in a patient with incomplete spinal cord injury, ${ }^{2}$ the signs and symptoms of $\mathrm{AD}$ generally include a sudden increase in blood pressure, altered heart rate (reflex bradycardia), anxiety, blurred vision, headache, flushing and sweating (above the level of injury). ${ }^{3}$ Bladder and bowel irritations are the most common causes of AD. Gastroenterologists need to be aware of the condition and typical HR-ARM findings compatible with $\mathrm{AD}$.

Received: March 3, 2014 Revised: March 10, 2014 Accepted: March 12, 2014

(c) This is an Open Access article distributed under the terms of the Creative Commons Attribution Non-Commercial License (http://creativecommons. org/licenses/by-nc/3.0) which permits unrestricted non-commercial use, distribution, and reproduction in any medium, provided the original work is properly cited.

*Correspondence: Joon Seong Lee, MD Institute for Digestive Research, Soonchunhyang University, College of Medicine, Daesagwan-gil 22, Yongsan-gu, Seoul 140-743, Korea.

Financial support: None. Tel: +82-2-709-9691, Fax: +82-2-709-9696, E-mail: joonlee@schmc.ac.kr

Conflicts of interest: None.

Author contributions: Tae Hee Lee designed the study and wrote the manuscript; Joon Seong Lee and Su Jin Hong reviewed the manuscript. 

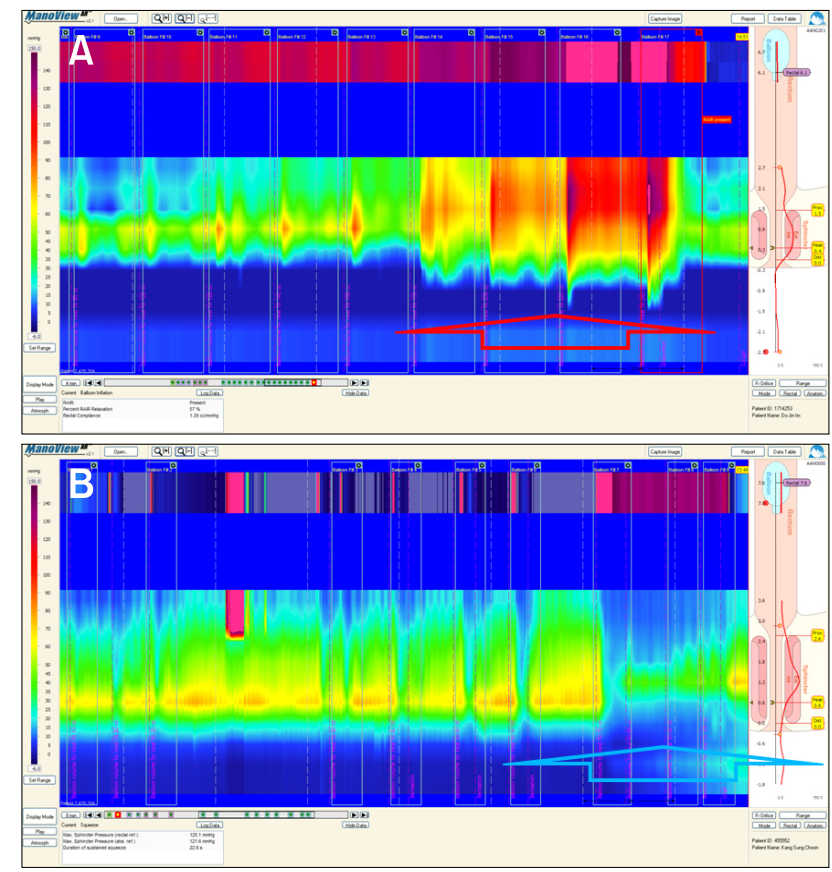

Figure. Effects of rectal distention in high-resolution anorectal manometry in a 48-year-old male with autonomic dysreflexia (A) compared to that in a 48-year-old female (B) without autonomic dysreflexia. During rectal distention, a patient with autonomic dysreflexia showed increasing degree of paradoxical anal contraction (red arrow), while a patient without autonomic dysreflexia showed increasing degree of anal relaxation (blue arrow).

\section{References}

1. Milligan J, Lee J, McMillan C, Klassen H. Autonomic dysreflexia: recognizing a common serious condition in patients with spinal cord injury. Can Fam Physician 2012;58:831-835.

2. Karlsson AK. Autonomic dysreflexia. Spinal Cord 1999;37:383-391.

3. Cragg J, Krassioukov A. Autonomic dysreflexia. CMAJ 2012;184:66. 